\title{
SINCRONIZAÇÃO DO CONHECIMENTO NO DESENVOLVIMENTO DE PRODUTO E MODELO DE NEGÓCIO: O CASO DA IMPRESSORA 3D
}

Antonio Batocchio (batocchi@fem.unicamp.br) - Materiais e Processos de Fabricação, UNICAMP

Samuel dos Santos Silva (189097@dac.unicamp.br) - Materiais e Processos de Fabricação, UNICAMP

Matheus Franco (matheusfranco400@gmail.com) - Materiais e Processos de Fabricação, UNICAMP

Vinicius Minatogawa (minatogawa@fem.unicamp.br) - Materiais e Processos de Fabricação, UNICAMP

Izabela Simon Rampasso (izarampasso@gmail.com) - Materiais e Processos de Fabricação, UNICAMP

Danilo Gusmão (danilogusmao@gmail.com) - Materiais e Processos de Fabricação, UNICAMP

\section{RESUMO}

O desenvolvimento de produtos, por vezes, é cercado de incertezas. Especialmente por seu sucesso estar atrelado não apenas a desafios técnicos, porém, também mercadológicos. Com a evolução da ciência de desenvolvimento de produtos e da competitividade empresarial, hodiernamente enxerga-se o mercado além das fronteiras da parte final de uma cadeia de suprimentos, não apenas como simples receptor de resultados. Isto é, o desenvolvimento de produtos passa pelo entendimento e compreensão das necessidades dos consumidores. Em investigação bibliográfica é possível verificar vasto arcabouço literário referente ao desenvolvimento de produtos, bem como de sua relação intrínseca com o mercado. Todavia, não é notória a presença de estudos que demonstram como ocorre a gestão do conhecimento, no intercâmbio de informações entre a criação do novo produto e o aprendizado sobre as necessidades do mercado. Desta forma, o presente trabalho procurou realizar simultaneamente o desenvolvimento de uma impressora 3D como produto, alinhado com informações do mercado e amparado por modelos de gestão do conhecimento. Como resultado observou-se informações mercadológicas influenciando nas decisões relacionadas ao desenvolvimento da impressora, e desafios técnicos alterando o desenvolvimento do modelo de negócio do produto, num contexto de gestão do conhecimento.

Palavras-chave: Desenvolvimento de Produtos; Desenvolvimento de Clientes; Gestão do Conhecimento

Área: Desenvolvimento de novos Produtos

\section{INTRODUÇÃO}

O objetivo do desenvolvimento de produtos é criar produtos que correspondam às necessidades dos consumidores. O sucesso de muitas empresas depende dessa habilidade de identificar necessidades de clientes, e introduzir a estes produtos que os satisfaçam (Mkrtchyan, 2015). Desta forma, o desenvolvimento de novos produtos pode ser entendido como um processo composto por um número de fases definidas (Cooper, 2008), e fundamentalmente multidisciplinar (Olson, 2001). Tais características fazem com que o processo de desenvolvimento ocorra com equipes de competências diversificadas como P\&D, projeto, engenharia, manufatura, marketing e compras (Mishra and Shah, 2009). O sucesso na 
implementação de novos produtos leva a empresa para um importante ganho em desempenho e fornece vantagem competitiva (Mobini, 2015).

O que se observa, portanto, é que o desenvolvimento de produto trata de transformar oportunidades de mercado para atender as necessidades dos consumidores e atingir as metas estratégicas da empresa (Cha 2014). De acordo com Rubera et al. (2016) desenvolver novos produtos é ligar tecnologia às necessidades dos clientes, o que requer conhecimento proveniente tanto do mercado quanto da tecnologia. Sendo assim, o desenvolvimento de produtos precisa ser orientado pelo consumidor. Isto é, entender que os sentimentos e necessidades do consumidor devem ser reconhecidos como algo de valor inestimável para fabricantes (Nagamachi, 2002).

Especialmente ao que tange o contexto de corporações, a literatura sobre o desenvolvimento de produtos é vasta (Krishnan et al 2001), e estudos para entendimento do mercado consumidor também apresentam um arcabouço bibliográfico relevante. Porém, em anos recentes, estudos focados em empresas nascentes de base tecnológicas (startups) passam a tratar o desenvolvimento de produtos de forma adaptada a este tipo de organização.

Uma abordagem diferenciada para estas empresas emergentes, em relação ao desenvolvimento de produtos, pode ser compreendida pela diferença de recursos. A criação e o surgimento desses empreendimentos emergentes é frequentemente um processo iterativo entre empreendedores e os recursos que dispõem (Alvarez and Barney, 2007; Alvarez et al., 2013; Sarasvathy, 2001). Mesmo em corporações já estabelecidas por vezes o desenvolvimento de produtos é conduzido de forma não sistemática e recursos são desperdiçados por falta de comunicação pelas diferentes partes envolvidas no desenvolvimento de produtos (Matzler et al. 1998).

Abordagens como a de Blank (2007) e Ries (2011) trouxeram ferramentas que propunham a minimização dos riscos do desenvolvimento de produtos para startups, pelas validações de hipóteses sobre o negócio paralelamente a concepção do produto. Isto é, para este tipo de abordagem, o desenvolvimento de produto deve estar acompanhando do desenvolvimento dos clientes (customer development). Desta maneira, haveria uma troca de conhecimento de mercado e produto que conduziria todo o projeto.

Entretanto, em pesquisa bibliográfica constatou-se que pouco são os estudos que abordam a gestão do conhecimento neste cenário. Ou seja, como ocorre a gestão do conhecimento, no intercâmbio de informações entre a criação do novo produto e o aprendizado sobre as necessidades do cliente, num contexto de empresas startup? A partir desta questão foi criada a hipótese de que a utilização do modelo de SECI (2008) para gestão de conhecimento, pode auxiliar por meio da transferência de conhecimento entre essas etapas ao longo de suas fases do framework.

Portanto, os objetivos deste trabalho se limitaram a:

- $\quad$ Aplicar práticas do customer development (Blank 2007) para entender as necessidades de determinado tipo de cliente.

- Iniciar o desenvolvimento de um produto com base nos aprendizados colhidos nas interações com os potenciais clientes, na fase de customer development.

- Utilizar o modelo de SECI (2008) para entender as iterações de conhecimento que ocorrem entre o desenvolvimento do produto e dos clientes. 


\section{REVISÃO BIBLIOGRAFICA}

\subsection{Desenvolvimento de Produto}

Desenvolvimento de produto pode ser entendido como a transformação da necessidade do mercado em um conjunto de suposições de um produto disponível para venda (Krishnan et al 2001). Ainda segundo Mirtalaie et. al, 2017, o Desenvolvimento de Produto é uma combinação de etapas que começa desde a identificação da necessidade do mercado até o lançamento do produto. O Processo de desenvolvimento de produto deve possuir equipes multidisciplinares como P\&D, engenharia, manufatura, marketing e compras (Mishra e Shah, 2009).

No entanto, no ambiente competitivo atual, as empresas que conseguem sobreviver são aquelas que conseguem responder rapidamente às necessidades do mercado, entregando produtos com qualidade e confiabilidade no tempo certo (Wang, et al, 2017). Gautam et al 2008, argumenta que uma empresa só consegue se manter competitiva se ganhar lucros e manter o cliente entusiasmado com seus produtos. A chave para a lucratividade das empresas é o desenvolvimento de novos produtos (Cooper, 2011).

De acordo com Mirtalaie et. al, 2017, as empresas além de manterem sua base de clientes, precisam desenvolver novos mercados para ganhar Market Share e uma maneira de se conseguir isto é através do desenvolvimento de novos produtos. No entanto, segundo Cooper 2011, na maioria das empresas, são raros os casos de sucesso no desenvolvimento de novos produtos.

Segundo Bijmolt et al 2010, as empresas falham no desenvolvimento de novos produtos, pois ainda não consideram o cliente como parte integrante processo de desenvolvimento, e assim, não envolvem o cliente ativamente neste processo. Com o envolvimento do cliente ao longo do processo de desenvolvimento do produto, não é necessário esperar até que o produto seja lançado, para saber se o cliente não estará disposto a pagar por aquilo, consegue-se descobrir isto durante o processo, evitando assim um grande desperdício de recursos como tempo e dinheiro (Alvarez, 2014).

Neste contexto, surge o Desenvolvimento de Clientes que vem sendo defendido e promovido por Blank (2007), entre outros como Blank e Dorf (2013), Cooper e Vlaskovits (2013), e Maurya (2012). Buscando dialogar com o cliente durante todo o processo de desenvolvimento de produto, em um processo de co-criação iterativo de aprendizado mútuo (Payne et al 2008, Ballantyne 2004) onde o cliente passa a ser parte ativa do processo (Payne et al 2008, Prahalad and Ramaswamy 2000).

\subsection{Desenvolvimento de Cliente (Customer Development)}

O Desenvolvimento de Clientes é um modelo iterativo composto de quatro etapas: Customer Discovery, onde o foco é entender os problemas e as necessidades dos clientes; Customer Validation, que é a identificação e validação da resolução do problema e possibilidade de captura de valor; Customer Creation, que é realmente gerar demanda para o cliente e descobrir possibilidades de escalar o negócio e Company Building, é a transição do seu produto validado para a criação de um negócio real, sempre mantendo este ciclo de aprendizado e descoberta de novos clientes e desenvolvimento de novos produtos (York e Danes 2014, Blank 2007). 


\section{Iteração}

\section{Execução}

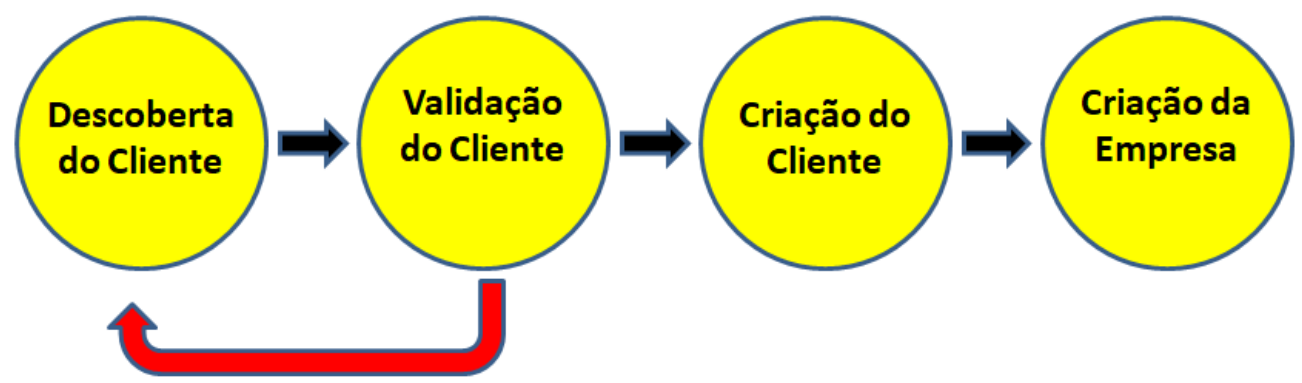

Desenvolvimento de Clientes é uma abordagem diferente da tradicional pesquisa de mercado. Enquanto a pesquisa de mercado busca entender as preferências e necessidades do cliente, o Desenvolvimento de Clientes vai muito além, utilizando um método científico para aprender com o cliente através de tentativa e erro (Cooper e Vlaskovits 2010, Alvarez 2014). Segundo York e Danes 2014, para que o Desenvolvimento de Clientes ocorra é necessário que haja um processo de contato direto entre empresa e cliente para aquisição de conhecimento e validação de hipóteses.

Segundo Blank 2013, os testes para validação com os clientes para desenvolvimento de produtos precisam ser rápidos e frequentes, sempre avaliando os resultados obtidos nesta iteração para adaptação e melhoria do produto. Este é um processo que nunca acaba, cada nova decisão envolvida deve ser antes validada com o cliente, melhorado o produto e testando novamente para receber os feedbacks.

Vantagem competitiva tem sido, crescentemente, mais associada à habilidade de a empresa aprender e melhorar de maneira contínua como parte da sua cultura. Aprender como engajar seus clientes e trazê-los mais próximo da empresa como um parceiro no desenvolvimento (Alvarez, 2014).

Segundo Payne et al 2008, o conhecimento sobre o cliente é um processo que deve ser baseado em dados e incorporados aos processos de desenvolvimento de novos produtos. A gestão do Conhecimento se torna de grande valia para gerenciar todo este processo de aprendizado do cliente, para que este conhecimento adquirido não se perca ao longo do processo de desenvolvimento e também para que se faça o uso adequado dos feedbacks recebidos pelos clientes no processo de Desenvolvimento de Clientes.

\subsection{Gestão do Conhecimento}

A Gestão do Conhecimento tornou-se fundamental para a geração da vantagem competitiva e sobrevivência das organizações no atual mercado, em que um grande número de tecnologias são criadas e outras tantas se tornam obsoletas diariamente, com uma grande demanda por lançamento de produtos.

O conhecimento é uma fonte de vantagem competitiva, uma vez que promove a inovação (Grant, 1996; Nonaka and Takeuchi, 1995). Sendo assim, o conhecimento fornecido pelos clientes podem ajudar as empresas a avaliar e satisfazer adequadamente as necessidades dos 
clientes e reduzir o risco de falha no desenvolvimento de produtos (Filieri 2013; Ogawa e Piller, 2006).

Em um mundo no qual mercados, produtos, tecnologias, competidores, normas e até mesmo sociedades mudam rapidamente, inovação contínua e os conhecimentos que permitem a inovação tornaram-se fontes importantes de vantagem competitiva (Nonaka et al., 2000). Segundo Nonaka, 1991 a criação de conhecimento ocorre conforme a espiral de criação dinâmica de conhecimento SECI - Socialização, Externalização, Combinação e Internacionalização. (Figura 2).

Figura 2. Espiral de Criação dinâmica de conhecimento SECI Fonte: Nonaka et al (2000)

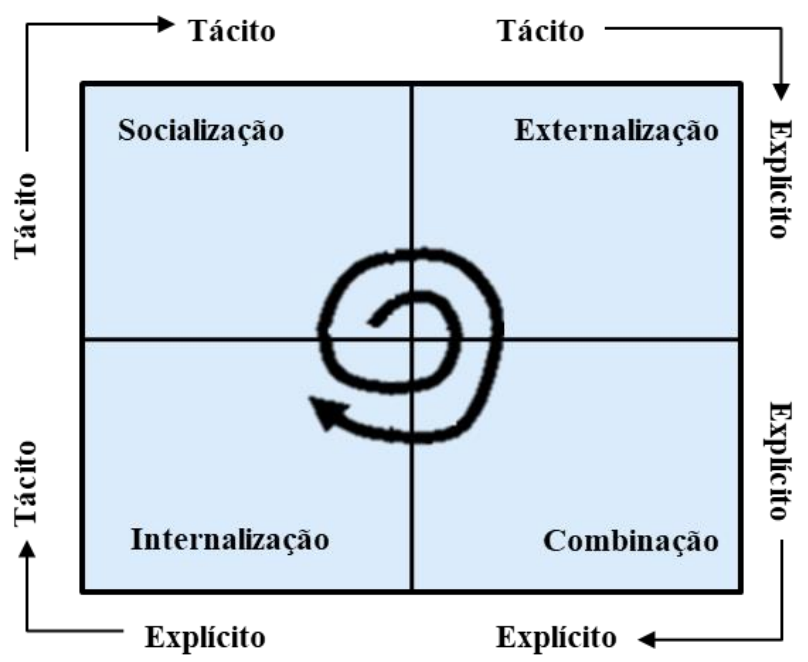

Alguns estudos foram realizados sobre como as empresas precisam aprender e aproveitar as competências e conhecimento dos clientes (Filieri 2013; Lilien et al 2002). No entanto poucos estudos se concentram em como os clientes são envolvidos no processo de desenvolvimento de produtos. Neste contexto, este trabalho tenta preencher estas lacunas através de um estudo de Pesquisa-ação (Action Research), descrevendo como ocorre o intercâmbio de informações entre a criação de um novo produto e o aprendizado sobre as necessidades do cliente. Foi então criada uma hipótese de que a utilização do modelo SECI (2000) para gestão do conhecimento, pode auxiliar por meio da transferência de conhecimento entre estas etapas ao longo de suas fases do framework.

\section{METODOLOGIA}

\subsection{Justificativa metodológica}

O método de estudo adotado foi o de Pesquisa-ação (Action Research) que segundo Huang 2010, é indicado para a criação de conhecimento em um contexto de prática e exige que os pesquisadores trabalhem como profissionais. De acordo com Cassel et al 2006, o uso da Pesquisa-ação (Action Research) vem sendo usado pelas organizações e pesquisadores para descrever e justificar as atividades onde o pesquisador é o agente da Mudança.

O que geralmente é visto como particularidade sobre a Pesquisa-ação (Action Research) é um ciclo iterativo de identificação de problemas, diagnóstico, planejamento, intervenção e avaliação dos resultados para aprender e planejar futuras intervenções (Checkland, 1991; Dickens et al 1999; Cassel et al 2006). Ao contrário das ciências sociais convencionais, seu objetivo não é unicamente compreender os arranjos sociais, mas também efetuar a mudança 
desejada como um caminho para gerar conhecimento e capacitar as partes interessadas (Huang, 2010).

O Método de Pesquisa-ação (Action Research) foi adotado ainda por ser uma abordagem para a resolução de problemas e experimentação prática, onde os resultados desejados da abordagem não são apenas soluções para os problemas imediatos, mas a aprendizagem importante a partir de resultados pouco explorados, e uma contribuição para o conhecimento científico (Coughlan, 2002).

\subsection{Contexto de aplicação}

Conforme o objetivo do trabalho, para que se pudesse conferir dados que partissem tanto do desenvolvimento de produto, quanto do "Desenvolvimento de Clientes", dentro de um contexto de pesquisa-ação, os pesquisadores deste projeto tomaram a decisão de desenvolver internamente um produto. Este contexto será considerado a unidade de análise do projeto.

Esta escolha se deu pelos seguintes fatores: (1) O fato de o produto ser criada no laboratório de Engenharia Mecânica da Unicamp, onde os pesquisadores do presente estudo trabalham ativamente, seguindo a metodologia de pesquisa-ação, o que facilitaria a obtenção dos dados para análise; (2) sendo os pesquisadores os criadores do produto, é possível a realização da Pesquisa-ação (Action Research) que de acordo com Huang 2010, se desenvolve em um contexto de prática e exige que os pesquisadores trabalhem como profissionais; (3) o fato de se trabalhar com impressão 3D, pois, segundo Rayna e Striukova 2016, é uma tecnologia que permite empreendedores potenciais testar e validar ideias rapidamente.

A metodologia adotada para obtenção dos dados baseou-se na metodologia desenvolvida por Kurt Lewin (1946), que combina a geração de teoria com a mudança no sistema social, e a maneira como ocorre a mudança no sistema e a geração de conhecimento. De acordo com Lewin (1946) são necessários quatro passos (Steps) que trabalham como um círculo contínuo de aprendizado: Planejamento, ação, observação e reflexão. Este trabalho foi realizado com 1 ciclo completo de pesquisa.

\section{RESULTADO E DISCUSSÃO}

\subsection{Planejamento}

O Planejamento é o estágio inicial do projeto de pesquisa no qual decidiu-se que as fontes de dados seriam entrevistas, observações e análise documental de forma a obter a triangulação dos dados. Foi desenvolvido um modelo baseado em dois modelos SECI em paralelo, um para desenvolvimento de clientes e outro para o de produtos. Por se tratar de uma ideia de aplicação da impressão 3D nunca testada, iniciou-se pelo ciclo de aprendizado do Desenvolvimento de Clientes, cujo conhecimento chave (C.C.) criado sobre o que fazer e para quem fazer será utilizado na etapa de engenharia simultânea do desenvolvimento de produtos que desdobrará em planos de ação, implementados com a aplicação de Scrum (metodologia ágil para gestão de projetos) para a geração de conhecimentos chave e/ou novos produtos conforme primeiras necessidades de mercado levantadas. Esse processo é realizado sucessivamente até a validação de produto e mercado. 
Figura 3: Framework para sincronização do Conhecimento entre Desenvolvimento de Clientes e Desenvolvimento de Produto baseado em dois ciclos SECI (Nonaka e Takeushi, 2000) paralelos , Fonte: Os autores

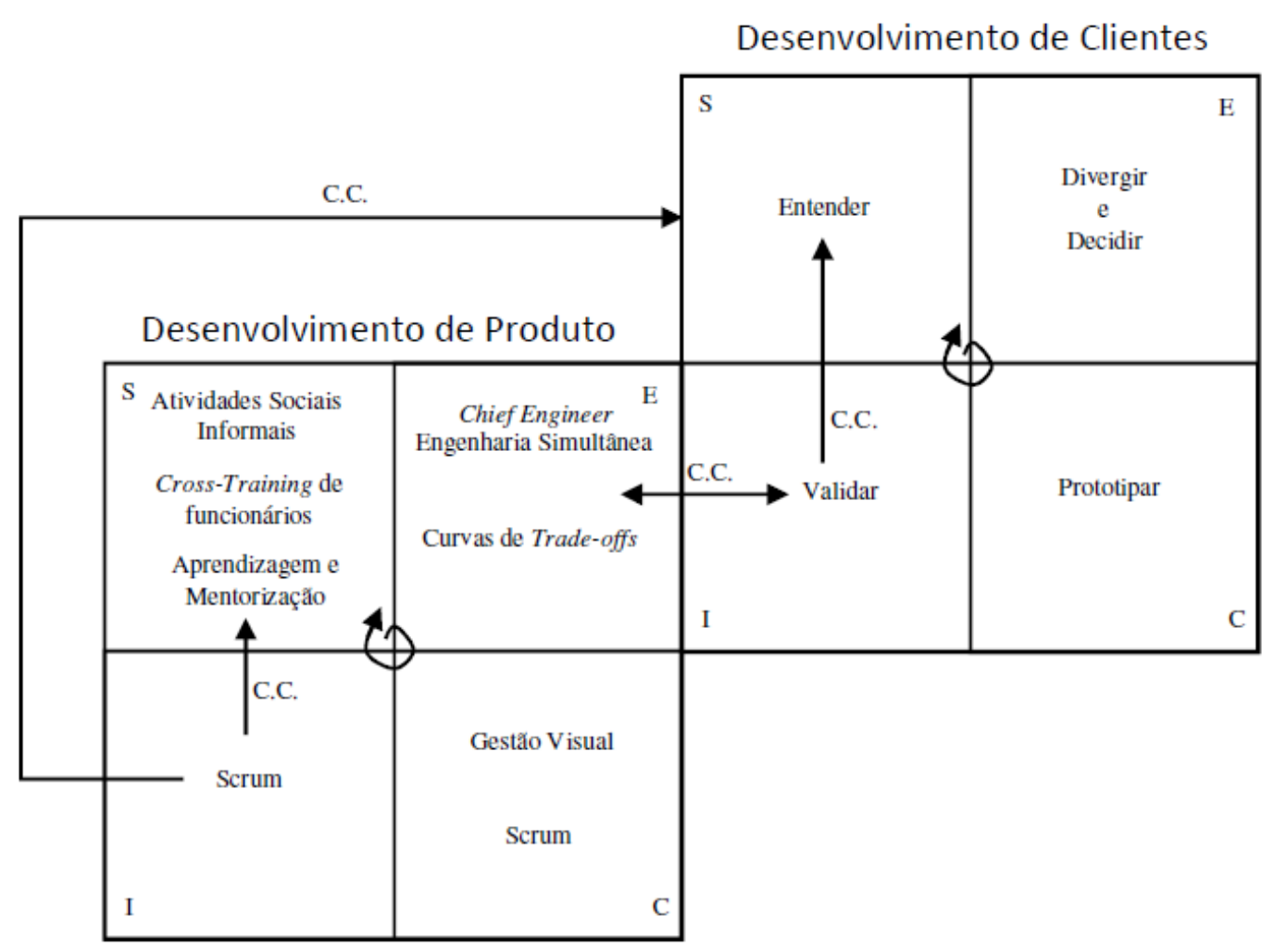

No quadrante do Desenvolvimento de Clientes existe quatro etapas dentro do contexto de socialização, externalização, combinação e internalização: Na Primeira etapa, entender, é realizado um estudo sobre as aplicações possíveis; na segunda etapa, Divergir e Decidir, diverge-se através de brainstorming com idéias que poderiam ser utilizadas como segmento de clientes de um modelo de negócio para, neste caso, a impressão $3 \mathrm{D}$, e decide-se qual a melhor idéia e, por fim, levanta-se de hipóteses para validação; na Prototipação cria-se algo para que possa ser apresentado ao cliente, para compreender as suas reações; finalmente, a quarta etapa é a validação, na qual é realizado a interação com o cliente, e coleta-se o feedback para criação de novo conhecimento que, caso positivo, inicia-se o processo de desenvolvimento do produto e caso negativo, outro ciclo é realizado com o teste de outras ideias.

Os conhecimentos chave gerados do processo anterior, são ativos a serem utilizados pelo desenvolvimento de produtos, a fim de minimizar custos e direcionar os esforços para de fato solucionar os problemas encontrados pelos clientes. Este quadrante também é composto de quatro etapas: a primeira etapa consiste no treinamento e aquisição de conhecimento prévio sobre o produto. Através de atividades Sociais Informais, Cross-Training de funcionários e aprendizagem e Mentorização; na segunda etapa recebe-se as informações provenientes do ciclo de desenvolvimento de clientes e o Chief Engineer é o responsável por traduzi-las para a linguagem de produto como engenharia simultânea. As curvas trade-offs são utilizadas para análise do custo benefício das informações recebidas dos clientes; os conhecimentos chave dos clientes, aliados ao conhecimento de produto, são desdobrados em planos de ação a serem realizados na terceira etapa através da gestão visual e scrum; da terceira para a quarta etapa é realizada a execução dos planos de ação, e cria-se conhecimento através do learn by doing. Os conhecimentos criados neste processo são utilizados como dados de entrada para ambos os desenvolvimentos de clientes e de produtos novamente em um ciclo virtuoso de aprendizado. 


\subsection{Ação}

De acordo com Lewin (1946), o passo da ação descreve como o estudo foi conduzido. Com base no Framework para sincronização do Conhecimento entre o Desenvolvimento do Cliente e Desenvolvimento Produto serão apresentados as etapas da aplicação do estudo.

\subsubsection{Aplicação do primeiro ciclo}

O processo iniciou-se pelo ciclo de desenvolvimento de cliente. As etapas de entender, divergir e decidir foram realizadas em paralelo. Durante esse processo de aprendizado e ideação (Brainstorming) buscou-se responder qual seria um segmento de clientes viável para aplicação da Tecnologia de impressão 3D. Dentre as ideias levantadas, optou-se pelo segmento de escolas, com a aplicação em educação infantil.

$\mathrm{Na}$ prototipação decidiu-se entrevistar pedagogas para validar se esta ideia tem possível impacto e, portanto, deve ser investigada. Realizou-se então entrevistas semi-estruturadas com seis pedagogas que trabalham ou trabalharam com educação infantil. Os feedbacks recebidos das pedagogas validaram a nossa primeira hipótese que a impressora 3D poderia ser utilizado no contexto de educação para melhoria no aprendizado infantil.

Em paralelo a esse ciclo, foi realizado também aprendizagem prévia sobre impressão 3D, as possíveis escolhas para aquisição com a análise de fornecedores, custos, tamanho e funcionamento. O Conhecimento adquirido com o Desenvolvimento do clientes, permitiu que a área de desenvolvimento do produto iniciasse o processo de aquisição das peças para a montagem da impressora 3D, pois, sabia-se que esta deveria ser pequena e com visual agradável. Optou-se por uma renomada fabricante Européia de Impressoras 3D com sede na cidade de Alicante na Espanha, como fornecedora das peças. Neste processo houve como resultado o aprendizado que não havia no Brasil, fornecedor qualificado que conseguisse fornecer as peças com baixo custo e em tempo hábil para a construção.

Durante o período de espera para a importação do material, e a fim de se obter mais conhecimentos chave sobre as necessidades e interesses dos clientes, iniciou-se um segundo ciclo de Desenvolvimento de Clientes. Isso pois, havia apenas conhecimento de que a aplicação seria de grande valia ao aprendizado, porém, seria necessário feedback dos diretores de escolas sobre a aplicabilidade, interesse, e possíveis necessidades relacionados ao uso.

\subsubsection{Aplicação do segundo ciclo}

O Segundo ciclo iniciou-se com o envio de email para vinte escolas localizadas na região de Piracicaba, das quais quatro responderam e uma delas agendou uma entrevista. Realizou-se então entrevista semi-estruturada com uma escola com mais de cinquenta anos de tradição que recebe quinhentos alunos entre os ensino infantil, fundamental e médio. A entrevista ocorreu com dois coordenadores pedagógicos e um diretor de ensino e vídeos do funcionamento da impressão 3D foram utilizados como protótipos. Nesta entrevista buscou-se entender de que forma a impressora 3D auxiliaria o processo de aprendizado do aluno e se a escola teria interesse no produto que estava sendo desenvolvido.

Os feedbacks recebidos e os conhecimentos criados neste segundo ciclo foram: a impressora 3D teria que funcionar com uma impressora tradicional, ou seja, o professor com apenas alguns clicks, sem dificuldades, conseguiria imprimir uma peça e sem a necessidade de funcionário especializado. Em outras palavras, deveria ser de fácil manuseio e com baixa manutenção.

Outro aprendizado que vale destacar que a impressora ajudaria no processo de aprendizado dos alunos, já que, segundo os coordenadores pedagógicos seria um instrumento essencial 
para transformar coisas abstratas em palpáveis e de fácil visualização. Como exemplo por estes citado, poderiam ser impressos os personagens das histórias para os alunos e, com isso, tornar a leitura muito mais interessante e prazerosa para eles e, consequentemente, melhorar o aprendizado já que este está diretamente ligado a atenção e interesse dos alunos.

Estes conhecimentos são importantes, pois, mesmo antes de a impressora ser montada, sabiase quais eram as principais características desejadas pelo cliente. Com isso, pôde-se continuar o processo de montagem da Impressora 3D, novamente no processo de desenvolvimento do produto, visando a obtenção de um produto de simples utilização, fácil manuseio e baixa necessidade de manutenção. No entanto, durante este processo encontrou-se problemas durante o desenvolvimento e, com isso, novos aprendizados e conhecimentos. Alguns exemplos são: não existe fornecedor do motor NEMA 14 (utilizado na montagem da impressora 3D) no Brasil e, portanto, tornou-se necessário importá-lo, o que aumentou o custo e o tempo consumidos; mesmo após a montagem, observou-se que a impressora necessitava de elevados tempos de setup entre cada impressão para nivelamento da mesa e limpeza do sistema de extrusão. Desta forma, pôde-se concluir que a ideia da impressora 3D ser implementada e manipulada nas escolas foi invalidada, inicialmente, devido a não adequação do produto às necessidades do cliente.

Com base neste conhecimento chave, optou-se que uma nova ideia seria manter a impressora fora das escolas, e fornecer apenas os materiais didáticos impressos. Neste novo ciclo que irá iniciar, busca-se, portanto, validar a hipótese supracitada a ser validada com o segmento de clientes para geração de novos conhecimentos.

\subsection{Observação}

Observou-se que a grande vantagem do processo de desenvolvimento de produto e cliente em paralelo, é o desenvolvimento de acordo a necessidade do cliente. Fato que corrobora com o argumento de Alvarez (2014) de que empresas grandes e pequenas estão buscando, cada vez mais, maneiras de manterem-se ágeis e inovadoras e para que isto ocorra é necessário uma abordagem diferente na forma de interagir com os clientes. Nesta linha de raciocínio, o envolvimento de times multidisciplinares atuando simultaneamente, proporcionou um aumento na troca de conhecimento entre as equipes e um melhor engajamento no desenvolvimento das atividades.

Outro ponto importante observado, foi que para entender realmente a real necessidade do cliente, é necessário considerar o cliente como parte do processo de desenvolvimento conforme argumentado por Bijmolt et al. (2010). Com o envolvimento do cliente ao longo do processo, percebeu-se que é possível evitar diversos desperdícios no processo de desenvolvimento como tempo e dinheiro além de canalizar os esforços conforme a necessidade, ou seja, de forma "puxada" de forma análoga com a filosofia Lean.

\subsection{Reflexão}

Como reflexão observou-se que a velocidade de lançamento de novos produtos tornou-se mais ágil a partir do momento que cliente foi envolvido no processo. Conforme proposto pelo framework de sincronização (figura 3), o aumento de agilidade acontece na sincronização de dois times trabalhando de forma integrada e simultânea, onde o conhecimento de um é transferido para o outro e assim sucessivamente. Este ciclo de aprendizado permite que os times se desenvolvam em um ciclo virtuoso de aprendizado. Notou-se também que com a sincronização, embora dificuldades foram encontradas durante o desenvolvimento de produtos, estas foram convertidas em conhecimentos chave para novo ciclo de Desenvolvimento de mercado. Resultado que corrobora com a visão de York e Danes 2014 de 
que precisa haver interação em todo o processo de desenvolvimento de produto para que haja a aquisição de conhecimento e validação de hipóteses. Este intercâmbio de conhecimento impede que o processo de desenvolvimento permaneça estagnado, o que está alinhado com a argumentação de Blank 2013, que diz que os testes para validação de hipóteses com os cliente permite o desenvolvimento de produto de forma mais ágil e eficaz.

\section{CONCLUSÃO}

É possível concluir com este trabalho que a aplicação do modelo SECI de criação e aquisição de conhecimento se mostrou eficaz no processo de desenvolvimento de novos produtos e desenvolvimento de mercado em paralelo. Conclui-se também que com a integração das atividades de Desenvolvimento de Produto e Mercado fazendo a troca de conhecimento, é possível obter novos produtos de forma mais rápida e eficaz que a tradicional,

Por fim, conclui-se que com o auxílio da metodologia de (Customer Development) para entender as necessidades dos clientes, foi possível validar ou invalidar hipóteses rapidamente em conversas com clientes, prevenindo assim o desperdício de tempo no desenvolvimento. $\mathrm{O}$ objetivo é a criação de produtos que atendam às reais necessidades dos clientes, já que não importa quão bonito ou inovador o seu produto seja, se os clientes não estiverem dispostos a pagar por ele, ele não tem valor algum. 


\section{REFERÊNCIAS}

ALVAREZ, C. Lean customer development: Building products your customers will buy. "O’Reilly Media, Inc.", 2014.

ARMISTEAD, C. Knowledge management and process performance. Journal of Knowledge Management, v. 3, n. 2, 143-157, 1999.

BALLANTYNE, D. Dialogue and its role in the development of relationship specific knowledge. Journal of Business \& Industrial Marketing, v. 19, n. 2, 114-123, 2004.

BIJMOLT, T.H.; LEEFLANG, P.S.; BLOCK, F.; EISENBEISS, M.; HARDIE, B.G.; LEMMENS, A. e SAFFERT, P. Analytics for customer engagement. Journal of Service Research, v. 13, n. 3, 341-356, 2010.

BLANK, S. The startup owner's manual: The step-by-step guide for building a great company. BookBaby, 2012.

BLANK, S. The four steps to the epiphany: successful strategies for products that win. BookBaby, 2013.

BRADBURY-HUANG, H. What is good action research? why the resurgent interest? Action Research, v. 8, n. 1, 93-109, 2010.

CASSELL, C. e JOHNSON, P. Action research: Explaining the diversity. Human relations, v. 59, n. 6, 783-814, 2006.

CHECKLAND, P. From framework through experience to learning: the essential nature of action research. Information System Research, 1991.

COOPER, B. e VLASKOVITS, P. The lean entrepreneur: How visionaries create products, innovate with new ventures, and disrupt markets. John Wiley \& Sons, 2013.

COOPER, R.G. e KLEINSCHMIDT, E.J. Newproducts: The key factors in success. Marketing Classics Press, 2011.

COUGHLAN, P. e COGHLAN, D. Action research for operations management. International jornal of operations \& production management, v. 22, n. 2, 220-240, 2002.

DICKENS, L. e WATKINS, K. Action research: rethinking lewin. Management Learning, v. 30, n. 2, 127-140, 1999.

FILIERI, R. Consumer co-creation and new product development: a case study in the food industry. Marketing Intelligence \& Planning, v. 31, n. 1, 40-53, 2013.

GAUTAM, N. e SINGH, N. Lean product development: Maximizing the customer perceived value through design change (redesign). International Journal of production economics, v. 114, n. 1, 313-332, 2008.

GRANT, R.M. Toward a knowledge-based theory of the firm. Strategic management journal, v. 17, n. S2, 109-122, 1996.

GUMMESSON, E. Qualitative methods in management research. Sage, 2000.

KOREN, Y.; WANG, W. e GU, X. Value creation through design for scalability of reconfigurable manufacturing systems. International Journal of Production Research, v. 55, n. 5, 1227-1242, 2017.

KRISHNAN, V. e ULRICH, K.T. Product development decisions: A review of the literature. Management science, v. 47, n. 1, 1-21, 2001. 
LEWIN, K. Action research and minority problems. Journal of social issues, v.2, n.4, 34-46, 1946.

LILIEN, G.L.; MORRISON, P.D.; SEARLS, K.; SONNACK, M. e HIPPEL, E.V. Performance assessment of the lead user idea-generation process for new product development. Management science, v. 48, n. 8, 1042-1059, 2002.

MAURYA, A. Running lean: iterate from plan A to a plan that works. "O’Reilly media, Inc.", 2012.

MIRTALAIE, M.A.; HUSSAIN, O.K.; CHANG, E. e HUSSAIN, F.K. A decision support framework for identifying novel ideas in new product development from cross-domain analysis. Information Systems, v. 69, 59-80, 2017.

MISHRA, A.A. e SHAH, R. In union lies strength: Collaborative competence in new product development and its performance effects. Journal of Operations Management, v. 27, n. 4, 324-338, 2009.

NONAKA, I. A empresa criadora de conhecimento. Harvard Business Review, v. 11, 1991.

NONAKA, I. e TAKEUCHI, H. The knowledge-creating company: How Japanese companies create the dynamics of innovation. Oxford university press, 1995.

OGAWA, S. e PILLER, F.T. Reducing the risks of new product development. MIT Sloan management review, v. 47, n. 2, 65, 2006.

PAYNE, A.F.; STORBACKA, K. e FROW, P. Managing the co-creation of value. Journal of the academy of marketing science, v. 36, n. 1, 83-96, 2008.

PRAHALAD, C.K. e RAMASWAMY, V. Co-opting customer competence. Harvard business review, v. 78, n. 1, 79-90, 2000.

RAYNA, T. e STRIUKOVA, L. From rapid prototyping to home fabrication: How 3d printing is changing business model innovation. Technological Forecasting and Social Change, v. 102, 214-224, 2016.

YORK, J.L. e DANES, J.E. Customer development, innovation, and decision-making biases in the lean startup. Journal of Small Business Strategy, v. 24, n. 2, 21, 2014. 